PROCEEDINGS OF THE

AMERICAN MATHEMATICAL SOCIETY

Volume 131, Number 4, Pages 1121-1129

S 0002-9939(02)06653-4

Article electronically published on June 12, 2002

\title{
BOREL SUBRINGS OF THE REALS
}

\author{
G. A. EDGAR AND CHRIS MILLER \\ (Communicated by David Preiss)
}

\begin{abstract}
A Borel (or even analytic) subring of $\mathbb{R}$ either has Hausdorff dimension 0 or is all of $\mathbb{R}$. Extensions of the method of proof yield (among other things) that any analytic subring of $\mathbb{C}$ having positive Hausdorff dimension is equal to either $\mathbb{R}$ or $\mathbb{C}$.
\end{abstract}

\section{Preliminaries}

We begin with a few definitions, notations, and results as preliminary material.

If $E$ is a set and $k$ is a positive integer, we write $E^{k}$ for the $k$-fold Cartesian product $E \times E \times \cdots \times E$.

The set $\mathbb{R}$ of real numbers has various structures: it is a field; it is a ring; it is a group (under addition); it is a metric space. The set $\mathbb{C}$ of complex numbers also has all these structures. Let $k$ be a positive integer. The Cartesian power $\mathbb{R}^{k}$ is a metric space, a group, and an $\mathbb{R}$-vector space, and $\mathbb{C}^{k}$ is a metric space, a group, a $\mathbb{C}$-vector space, and an $\mathbb{R}$-vector space.

If $X$ is a metric space and $A \subseteq X$, then $A$ is called a Borel set in $X$ iff $A$ belongs to the $\sigma$-algebra generated by the open sets in $X$. If $X$ and $Y$ are metric spaces, then a function $\varphi: X \rightarrow Y$ is called Borel measurable iff for every Borel set $B$ in $Y$, the inverse image $\varphi^{-1}(B)$ is a Borel set in $X$. If $X$ is a complete separable metric space, and $A \subseteq X$, then $A$ is called an analytic set in $X$ if $A$ is the continuous image of some Borel set in some Euclidean space $\mathbb{R}^{k}$. (Analytic sets are also known as Suslin sets or Souslin sets.)

We write dim for Hausdorff dimension. The Dimension Inequality 15. Thm. 8.10, p. 115], [10, 7.2, p. 94]: if $A \subseteq \mathbb{R}^{n}, B \subseteq \mathbb{R}^{m}$ are Borel sets, then $\operatorname{dim}(A \times B) \geq$ $\operatorname{dim} A+\operatorname{dim} B$. In general, equality need not hold. From this we get

$$
\operatorname{dim}\left(A^{n+m}\right) \geq \operatorname{dim}\left(A^{n}\right)+\operatorname{dim}\left(A^{m}\right) .
$$

Therefore the sequence $(1 / n) \operatorname{dim}\left(A^{n}\right)$ converges as $n \rightarrow \infty$ to $\sup _{n}(1 / n) \operatorname{dim}\left(A^{n}\right)$. Call this limit the Cartesian-Hausdorff dimension of $A$, or the $\mathbf{C H}$ dimension of $A$. All we need to know is that the $\mathrm{CH}$ dimension of $A$ is 0 iff $\operatorname{dim}\left(A^{n}\right)=0$ for all positive integers $n$. This implies in particular that $\operatorname{dim} A=0$.

Received by the editors October 29, 2001.

2000 Mathematics Subject Classification. Primary 28A78; Secondary 03E15, 11K55, 12D99, $28 \mathrm{~A} 05$.

Key words and phrases. Borel subring, Borel subfield, Hausdorff dimension, Erdős, Volkmann, Suslin sets, analytic sets.

Research of the second author was supported by NSF grant no. DMS-9988855.

(C)2002 American Mathematical Society 


\section{MAIN THEOREM}

We improve the main result of our paper [6], using some of the same methods for the proof.

Theorem 1. Let $E \subseteq \mathbb{R}$ be a subring and a Borel set. Then either $E$ has $C H$ dimension zero or $E=\mathbb{R}$.

Historical remarks. In 1960, Volkmann [17] noted that all known examples of subfields of $\mathbb{R}$ had Hausdorff dimension either 0 or 1 . He showed that if a subfield $K \subseteq \mathbb{R}$ existed with $\operatorname{dim} K=s, 0<s<1$, then it would be "dimensionslos"-for every open set $U$, the $s$-dimensional Hausdorff measure $\mathcal{H}^{s}(K \cap U)$ is either 0 or $\infty$. In 1966, Erdős and Volkmann 7] showed that for every $s, 0 \leq s \leq 1$, there is $G \subseteq \mathbb{R}$ an additive subgroup and a Borel set, with $\operatorname{dim} G=s$. (See also 10 . Ex. 12.4, p. 167].) They noted that the question of the Hausdorff dimension of subrings and subfields remained open. R. O. Davies (unpublished, see 9. p. 212] and [15, p. 167]), using the Continuum Hypothesis, showed that for $0 \leq s \leq 1$, there exists a subring $E \subseteq \mathbb{R}$ with $\operatorname{dim} E=s$. These subrings are not Borel (and not analytic). In 1984, Falconer [8] showed that if $E \subseteq \mathbb{R}$ is a subring and a Borel set (or an analytic set), then $\operatorname{dim} E \leq 1 / 2$ or $\operatorname{dim} E=1$. He gave a second proof in [9]. The authors [6] recently showed that if $K \subseteq \mathbb{R}$ is a real-closed subfield and a Borel set (or an analytic set), then either $\operatorname{dim} K=0$ or $K=\mathbb{R}$.

The proof of Theorem 1 will be subdivided into lemmas.

The set of linear functionals on $\mathbb{R}^{k}$ is naturally identified with $\mathbb{R}^{k}$. When we say "almost all" linear functionals, we mean almost all with respect to $k$-dimensional Lebesgue measure.

Lemma 1.1. Let $A \subseteq \mathbb{R}^{k}$ be a Borel set with $\operatorname{dim} A>1$. Then for almost all linear functionals $\varphi: \mathbb{R}^{k} \rightarrow \mathbb{R}$, the image $\varphi(A)$ has positive Lebesgue measure.

Proof. This is a special case of the Projection Theorem [15, Cor. 9.8, p. 131]. A proof for $k=2$ may be found in [10, Thm. 6.1, p. 83]. Note that the image $\varphi(A)$, although it may not be Borel, is analytic, and therefore Lebesgue measurable [3]. Thm. 8.4.1, p. 278].

Lemma 1.2. Let $E \subseteq \mathbb{R}$ be an additive subgroup and a Borel set with nonzero $C H$ dimension. Then there is a positive integer $k$ and a linear functional $\varphi: \mathbb{R}^{k} \rightarrow \mathbb{R}$ such that $\varphi\left(E^{k}\right)=\mathbb{R}$.

Proof ([6]). Since $E$ has nonzero CH dimension, there is $n$ so that $\operatorname{dim}\left(E^{n}\right)>0$. So (by the Dimension Inequality) there is $k$ with $\operatorname{dim}\left(E^{k}\right)>1$. By Lemma 1.1. there is a linear functional $\varphi: \mathbb{R}^{k} \rightarrow \mathbb{R}$ such that $\varphi\left(E^{k}\right)$ has positive Lebesgue measure. Since $\varphi$ is linear, $\varphi\left(E^{k}\right)$ is an additive subgroup of $\mathbb{R}$. Now $\varphi\left(E^{k}\right)$ has positive measure, so by Steinhaus's Theorem ([1, Thm. 15.12, p. 116], [15, Ex. 4, p. 43], [16, (6.67), p. 297]), the difference set $\varphi\left(E^{k}\right)-\varphi\left(E^{k}\right)$ contains a neighborhood of 0 . But since $\varphi\left(E^{k}\right)$ is a subgroup, it is equal to its difference set. Every element of $\mathbb{R}$ is an integer multiple of an element of the neighborhood $\varphi\left(E^{k}\right)$ of zero. But then since it is a group, $\mathbb{R}=\varphi\left(E^{k}\right)$.

Lemma 1.3. Let $E \subseteq \mathbb{R}$ be a subring. Assume there is a positive integer $k$ and a linear functional $\varphi: \mathbb{R}^{k} \rightarrow \mathbb{R}$ such that $\varphi\left(E^{k}\right)=\mathbb{R}$. Then such $k$ and $\varphi$ may be found so that $\varphi$ also maps $E^{k}$ bijectively onto $\mathbb{R}$. 
Proof. Let $k$ be the least positive integer such that there is a linear functional $\varphi: \mathbb{R}^{k} \rightarrow \mathbb{R}$ with $\varphi\left(E^{k}\right)=\mathbb{R}$. We claim that $\varphi$ is injective on $E^{k}$. For $1 \leq j \leq k$, let $\mathbf{u}_{j}=(0, \cdots, 0,1,0, \cdots, 0)$ with 1 in the $j$ th coordinate, and write $r_{j}=\varphi\left(\mathbf{u}_{j}\right)$. The map $\varphi$ is $\mathbb{R}$-linear, so $\varphi\left(E^{k}\right)=\mathbb{R}$ means

$$
\left\{\sum_{j=1}^{k} a_{j} r_{j}: a_{1}, \cdots, a_{k} \in E\right\}=\mathbb{R} .
$$

Assume $\varphi$ is not injective on $E^{k}$. We will show that one of the $r_{j}$ may be dropped, while retaining the equality (因). Since $\varphi$ is not injective, there are $b_{1}, \cdots, b_{k} \in E$, not all zero, so that $\sum b_{j} r_{j}=0$. By re-labeling, if necessary, we may assume $b_{k} \neq 0$. So

$$
r_{k}=\sum_{j=1}^{k-1} \frac{-b_{j}}{b_{k}} r_{j}
$$

We claim

$$
\left\{\sum_{j=1}^{k-1} a_{j} r_{j}: a_{1}, \cdots, a_{k-1} \in E\right\}=\mathbb{R} .
$$

Let $s$ be any real number. Then $s / b_{k} \in \mathbb{R}$, so by (団) there exist $a_{1}, \cdots, a_{k} \in E$ such that $s / b_{k}=\sum_{j=1}^{k} a_{j} r_{j}$. Therefore

$$
s=\sum_{j=1}^{k-1} b_{k} a_{j} r_{j}+b_{k} a_{k} \sum_{j=1}^{k-1} \frac{-b_{j}}{b_{k}} r_{j}=\sum_{j=1}^{k-1}\left(b_{k} a_{j}-a_{k} b_{j}\right) r_{j} .
$$

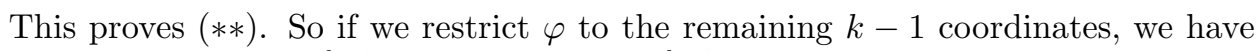
a linear functional $\mathbb{R}^{k-1} \rightarrow \mathbb{R}$ that maps $E^{k-1}$ onto all of $\mathbb{R}$. This contradicts the minimality of $k$. So $\varphi$ is injective on $E^{k}$.

Lemma 1.4. Let $E \subseteq \mathbb{R}$ be an additive subgroup and a Borel set. Let $k$ be a positive integer and $\varphi: \mathbb{R}^{k} \rightarrow \mathbb{R}$ a linear functional. Assume that $\varphi$ maps $E^{k}$ bijectively onto $\mathbb{R}$. Then $k=1$ and $E=\mathbb{R}$.

Proof. Since $\varphi$ is $\mathbb{R}$-linear, it is continuous, hence Borel measurable. Let $\psi: \mathbb{R} \rightarrow$ $E^{k}$ be the inverse of the restriction of $\varphi$ to $E^{k}$. Then $\psi$ is Borel measurable [3. Prop. 8.3.5, p. 274], 14, (15.2), p. 89] and a group homomorphism. For $1 \leq j \leq k$, let $\mathbf{u}_{j}=(0, \cdots, 0,1,0, \cdots, 0)$ with 1 in the $j$ th coordinate, and write $r_{j}=\varphi\left(\mathbf{u}_{j}\right)$. Let $\pi_{1}: \mathbb{R}^{k} \rightarrow \mathbb{R}$ be the first coordinate map. Then $\tau=\pi_{1} \circ \psi$ maps $\mathbb{R} \rightarrow \mathbb{R}$, $\tau(x+y)=\tau(x)+\tau(y)$ for all $x, y$, and $\tau$ is Borel measurable. Therefore there is a constant $c$ such that for all $x \in \mathbb{R}, \tau(x)=c x([1$, Ex. 11, p. 117], 16, p. 307, Ex. 9(e)], 14, (9.10), p. 61]). Now $\tau\left(r_{1}\right) \neq 0$, so $c \neq 0$; but if $k>1$, then there would be $r_{2} \neq 0$ with $\tau\left(r_{2}\right)=0$, a contradiction. Therefore $k=1$, so the linear functional $\varphi: \mathbb{R} \rightarrow \mathbb{R}$ has the form $\varphi(x)=a x$ for some constant $a$. But $\varphi$ maps $E$ onto all of $\mathbb{R}$, so $E=\mathbb{R}$.

Proof of Theorem 1 , Let $E \subseteq \mathbb{R}$ be a subring and a Borel set with nonzero Cartesian-Hausdorff dimension. Then $k$ and $\varphi$ exist as in Lemma 1.2 By Lemma 1.3 we may assume $\varphi$ maps $E^{k}$ bijectively onto $\mathbb{R}$. Then by Lemma 1.4, $E=\mathbb{R}$. 
Remarks. We have stated Theorem 1 for Borel sets. It is true for analytic sets as well:

(i) The Dimension Inequality $\operatorname{dim}(A \times B) \geq \operatorname{dim} A+\operatorname{dim} B$ is stated in [15] only for Borel sets. But it is also true for analytic sets. Given analytic sets $A, B$, there are compact sets $A_{1} \subseteq A$ and $B_{1} \subseteq B$ with Hausdorff dimension as close as we like to $\operatorname{dim} A, \operatorname{dim} B$ (4]; there is a proof in [5, (1.7.11), p. 62]). Apply the Borel set result to $A_{1} \times B_{1}$. (Actually $\operatorname{dim}(A \times B) \geq \operatorname{dim} A+\operatorname{dim} B$ is true for arbitrary sets, but with a more difficult proof [18, [13].)

(ii) The Projection Theorem (Lemma 1.1). If $A \subseteq \mathbb{R}^{k}$ is an analytic set and $\operatorname{dim} A>1$, choose a compact set $A_{1} \subseteq A$ with $\operatorname{dim} A_{1}>1$, and apply the Borel version of the theorem.

(iii) Borel isomorphism. If $A, B$ are analytic sets and $\varphi: A \rightarrow B$ is Borel measurable and bijective, then $\varphi^{-1}$ is also Borel measurable [3, Prop. 8.6.2, p. 289]. This (even the Borel version of it) is proved from the Separation Theorem for analytic sets [3, Thm. 8.3.1, p. 272].

If $X \subseteq \mathbb{R}$ is an analytic set, then the ring generated by it, $\mathbb{Z}[X]$, is an analytic set. (This shows one of the reasons why we are interested in the analytic case: the ring generated by a Borel set is not necessarily Borel.) If $\operatorname{dim} X^{k}>0$ for some $k$, then by our results $\mathbb{Z}[X]=\mathbb{R}$ : every real is a finite sum of finite products of elements of $X$ or their negatives. If $X$ is also compact, we may use the Baire Category Theorem on this. There is $n$ and an open interval $I$, so that every element of $I$ is a sum of at most $n$ terms, each of which is (plus or minus) a product of at most $n$ elements of $X$. (Empty sum is 0 , empty product is 1 .)

The groups of Erdős and Volkmann are not divisible (it is easy to check that $e$ belongs to all of them, but $e / 2$ belongs to none of them). One might ask whether a divisible additive subgroup of $\mathbb{R}$ (a vector space over $\mathbb{Q}$ ) can have Hausdorff dimension strictly between 0 and 1 . This can, indeed, happen. If $G$ is any additive subgroup that is Borel, then its divisible hull

$$
\bigcup_{n=1}^{\infty} \frac{1}{n !} G
$$

is a divisible additive subgroup of $\mathbb{R}$, is still a Borel set, and has the same Hausdorff dimension as $G$. Apply this observation to the Erdős-Volkmann groups.

\section{Subrings OF THE COMPLEX NUMBERS}

The method above may be adapted to prove similar results.

Theorem 2. Let $E \subseteq \mathbb{C}$ be a subring and a Borel set. Then $E$ has zero $C H$ dimension or $E=\mathbb{R}$ or $E=\mathbb{C}$.

The proof will use lemmas analogous to the lemmas for Theorem 1.

We will need a special case of the complex version of the Projection Theorem (which is proved in the same way as the real Projection Theorem, using the complex Grassmannian in place of the real Grassmannian). Since we did not find it in print, we include here the special case (1-complex-dimensional range) that is to be used in this paper. We follow the proof given by Mattila [15] for $\mathbb{R}$.

Lemma 2.1. Let $A \subseteq \mathbb{C}^{k}$ be a Borel set with $\operatorname{dim} A>2$. Then for almost all $\mathbb{C}$-linear functionals $\varphi: \mathbb{C}^{\bar{k}} \rightarrow \mathbb{C}$, the image $\varphi(A)$ has positive 2-dimensional Lebesgue measure. 
Proof. Define the inner product on $\mathbb{C}^{k}$ by: if $\mathbf{u}=\left(u_{1}, \cdots, u_{k}\right), \mathbf{v}=\left(v_{1}, \cdots, v_{k}\right)$, let

$$
\langle\mathbf{u}, \mathbf{v}\rangle=\sum_{j=1}^{k} u_{j} \overline{v_{j}} .
$$

Each fixed $\mathbf{v} \in \mathbb{C}^{k}$ induces a linear functional $\varphi_{\mathbf{v}}$ by $\varphi_{\mathbf{v}}(\mathbf{u})=\langle\mathbf{u}, \mathbf{v}\rangle$. All linear functionals are of this form. The norm for $\mathbb{C}^{k}$ is $\|\mathbf{u}\|=\sqrt{\langle\mathbf{u}, \mathbf{u}\rangle}$.

A unitary operator is a linear map $T: \mathbb{C}^{k} \rightarrow \mathbb{C}^{k}$ such that $\|T(\mathbf{u})\|=\|\mathbf{u}\|$ for all $\mathbf{u} \in \mathbb{C}^{k}$. Unitary maps on $\mathbb{C}^{k}$ may be identified with $k \times k$ unitary matrices. There is a transitivity property: if $\mathbf{u}, \mathbf{v} \in \mathbb{C}^{k}$ and $\|\mathbf{u}\|=\|\mathbf{v}\|$, then there is a unitary $T$ such that $T(\mathbf{u})=\mathbf{v}$.

In $\mathbb{C}^{k}$, the unit sphere $S=S^{2 k-1}$ is $S=\left\{\mathbf{u} \in \mathbb{C}^{k}:\|\mathbf{u}\|=1\right\}$. Write $\sigma$ for the $(2 k-1)$-dimensional surface area on $S$. Sometimes we act as though $\sigma$ is a measure on all of $\mathbb{C}^{k}$ by letting the complement of $S$ have measure zero. Note that $\sigma$ is invariant under unitary transformation: if $H \subseteq \mathbb{C}^{k}$ and $T$ is unitary, then $\sigma(T(H))=\sigma(H)$.

Write $\mathbf{u}_{1}=(1,0, \cdots, 0)$ for a "north pole" of $S$. A calculus exercise shows us that a band around the "equator" of $S$ has area $\sigma\left\{\mathbf{v} \in S:\left|\left\langle\mathbf{v}, \mathbf{u}_{1}\right\rangle\right| \leq r\right\} \leq \pi r^{2} C_{2 k-3}$, where $C_{2 k-3}$ is the $(2 k-3)$-dimensional volume of the unit $(2 k-3)$-ball. By the transitivity and invariance, for any $\mathbf{y} \in S$,

$$
\sigma\{\mathbf{v} \in S:|\langle\mathbf{v}, \mathbf{y}\rangle| \leq r\}=\sigma\left\{\mathbf{v} \in S:\left|\left\langle\mathbf{v}, \mathbf{u}_{1}\right\rangle\right| \leq r\right\} \leq \pi r^{2} C_{2 k-3},
$$

and so for any nonzero $\mathbf{y} \in \mathbb{C}^{k}$,

$$
\sigma\{\mathbf{v} \in S:|\langle\mathbf{v}, \mathbf{y}\rangle| \leq r\}=\sigma\left\{\mathbf{v} \in S:\left|\left\langle\mathbf{v}, \frac{\mathbf{y}}{\|\mathbf{y}\|}\right\rangle\right| \leq \frac{r}{\|\mathbf{y}\|}\right\} \leq \pi C_{2 k-3}\left(\frac{r}{\|\mathbf{y}\|}\right)^{2} .
$$

(This inequality corresponds to Mattila's [15, Lemma 3.11, p. 50], and will be used in the same way.)

Now let $A \subseteq \mathbb{C}^{k}$ be a Borel set with $\operatorname{dim} A>2$. Then by [5, (3.2.7), p. 121] there is a probability measure $\mu$ with compact support $\subseteq A$ so that

$$
I^{2}(\mu)=\iint \frac{\mu(d \mathbf{x}) \mu(d \mathbf{y})}{\|\mathbf{x}-\mathbf{y}\|^{2}}<\infty .
$$

For $\mathbf{v} \in S$, let $\mu_{\mathbf{v}}$ be the image of $\mu$ under the linear functional $\varphi_{\mathbf{v}}$, defined as follows: for $H \subseteq \mathbb{C}$, let $\mu_{\mathbf{v}}(H)=\mu\left\{\mathbf{x} \in \mathbb{C}^{k}:\langle\mathbf{x}, \mathbf{v}\rangle \in H\right\}$. So if $\mathbf{v} \in S, t \in \mathbb{C}$, and $r>0$, the measure of a disk in the complex plane is

$$
\mu_{\mathbf{v}}\{z \in \mathbb{C}:|z-t| \leq r\}=\mu\left\{\mathbf{x} \in \mathbb{C}^{k}:|\langle\mathbf{x}, \mathbf{v}\rangle-t| \leq r\right\} .
$$

Of course the 2-dimensional Lebesgue measure of a disk is

$$
\lambda^{2}\{z \in \mathbb{C}:|z-t| \leq r\}=\pi r^{2} .
$$

Therefore the lower density of $\mu_{\mathbf{v}}$ with respect to $\lambda^{2}$ is

$$
\begin{aligned}
\underline{D}\left(\mu_{\mathbf{v}}, \lambda^{2}, t\right) & =\liminf _{r \rightarrow 0} \frac{\mu_{\mathbf{v}}\{z \in \mathbb{C}:|z-t| \leq r\}}{\lambda^{2}\{z \in \mathbb{C}:|z-t| \leq r\}} \\
& =\liminf _{r \rightarrow 0} \frac{\mu\left\{\mathbf{x} \in \mathbb{C}^{k}:|\langle\mathbf{x}, \mathbf{v}\rangle-t| \leq r\right\}}{\pi r^{2}} .
\end{aligned}
$$


Now imitate the proof of [15, Thm. 9.7, p. 130]:

$$
\begin{aligned}
\iint \underline{D}\left(\mu_{\mathbf{v}}, \lambda^{2}, t\right) & \mu_{\mathbf{v}}(d t) \sigma(d \mathbf{v}) \\
& \leq \liminf _{r \rightarrow 0} \frac{1}{\pi r^{2}} \iint \mu\{\mathbf{x}:|\langle\mathbf{x}, \mathbf{v}\rangle-t| \leq r\} \mu_{\mathbf{v}}(d t) \sigma(d \mathbf{v}) \\
& =\liminf _{r \rightarrow 0} \frac{1}{\pi r^{2}} \iint \mu\{\mathbf{x}:|\langle\mathbf{x}-\mathbf{y}, \mathbf{v}\rangle| \leq r\} \mu(d \mathbf{y}) \sigma(d \mathbf{v}) \\
& =\liminf _{r \rightarrow 0} \frac{1}{\pi r^{2}} \iint \sigma\{\mathbf{v}:|\langle\mathbf{x}-\mathbf{y}, \mathbf{v}\rangle| \leq r\} \mu(d \mathbf{y}) \mu(d \mathbf{x}) \\
& \leq C_{2 k-3} \iint \frac{1}{\|\mathbf{x}-\mathbf{y}\|^{2}} \mu(d \mathbf{y}) \mu(d \mathbf{x}) \\
& =C_{2 k-3} I^{2}(\mu)<\infty .
\end{aligned}
$$

So, for $\sigma$-almost all $\mathbf{v}, \underline{D}\left(\mu_{\mathbf{v}}, \lambda^{2}, t\right)<\infty$ for $\mu_{\mathbf{v}}$-almost all $t$. By [15, 2.12(3), p. 36] for any such $\mathbf{v}$ we conclude $\mu_{\mathbf{v}} \ll \lambda^{2}$.

Finally, for any $\mathbf{v}$ with $\mu_{\mathbf{v}} \ll \lambda^{2}$, we claim $\lambda^{2}\left(\varphi_{\mathbf{v}}(A)\right)>0$. Indeed, if $\lambda^{2}\left(\varphi_{\mathbf{v}}(A)\right)$ $=0$, then $\mu_{\mathbf{v}}\left(\varphi_{\mathbf{v}}(A)\right)=0$, so

$$
0=\mu_{\mathbf{v}}\left(\varphi_{\mathbf{v}}(A)\right)=\mu\left\{\mathbf{x}: \varphi_{\mathbf{v}}(\mathbf{x}) \in \varphi_{\mathbf{v}}(A)\right\} \geq \mu(A)=1,
$$

a contradiction.

Lemma 2.2. Let $E \subseteq \mathbb{C}$ be an additive subgroup and a Borel set with nonzero $\mathrm{CH}$ dimension. Then there is a positive integer $k$ and a complex-linear functional $\varphi: \mathbb{C}^{k} \rightarrow \mathbb{C}$ such that $\varphi\left(E^{k}\right)=\mathbb{C}$.

Proof. Since $E$ has nonzero $\mathrm{CH}$ dimension, there is $n$ so that $\operatorname{dim}\left(E^{n}\right)>0$. So (by the Dimension Inequality) there is $k$ with $\operatorname{dim}\left(E^{k}\right)>2$. By Lemma 2.1, there is a $\mathbb{C}$-linear functional $\varphi: \mathbb{C}^{k} \rightarrow \mathbb{C}$ such that $\varphi\left(E^{k}\right)$ has positive 2-dimensional Lebesgue measure. The rest is the same as before. We need the 2-dimensional version of Steinhaus's Theorem (due to Ruziewicz): $\varphi\left(E^{k}\right)$ has positive measure, so $\varphi\left(E^{k}\right)-\varphi\left(E^{k}\right)$ contains a neighborhood of 0 ([1 Thm. 15.12, p. 116], [15, Ex. 4, p. 43]).

Lemma 2.3. Let $E \subseteq \mathbb{C}$ be a subring. Assume there is a positive integer $k$ and $a$ complex-linear functional $\varphi: \mathbb{C}^{k} \rightarrow \mathbb{C}$ such that $\varphi\left(E^{k}\right)=\mathbb{C}$. Then such $k$ and $\varphi$ may be found so that $\varphi$ also maps $E^{k}$ bijectively onto $\mathbb{C}$.

Proof. The proof is as before, using $\mathbb{C}$-linear instead of $\mathbb{R}$-linear.

Lemma 2.4. Let $E \subseteq \mathbb{C}$ be an additive subgroup and a Borel set. Let $k$ be a positive integer and $\varphi: \mathbb{C}^{k} \rightarrow \mathbb{C}$ a $\mathbb{C}$-linear functional. Assume that $\varphi$ maps $E^{k}$ bijectively onto $\mathbb{C}$. Then either $k=1$ and $E=\mathbb{C}$ or $k=2$ and $E=\mathbb{R}$.

Proof. Let $\psi: \mathbb{C} \rightarrow E^{k}$ be the inverse of the restriction of $\varphi$. Let $\mathbf{u}_{j}, r_{j}=\varphi\left(\mathbf{u}_{j}\right)$ as before. Then $\tau=\pi_{1} \circ \psi$ maps $\mathbb{C} \rightarrow \mathbb{C}$, is additive, and is Borel measurable. Therefore it is continuous. (See [2 Ch. I, Thm. 4], [14, (9.10), p. 61].) Now $\tau$ is $\mathbb{R}$-linear, so its nullspace could be:

(1) the single point 0 ;

(2) a 1-real-dimensional line through 0 ; or

(3) all of $\mathbb{C}$. 
In case (1), we conclude $k=1$ as before and therefore $E=\mathbb{C}$. Case (3) is ruled out since $S\left(r_{1}\right)=1 \neq 0$. So in the case where $E \neq \mathbb{C}$ we have case (2). This means $\left\{r_{2}, \cdots, r_{k}\right\}$ all lie in this one line through 0 . Repeating with the other coordinates, we conclude $k=2$ and $r_{1}, r_{2}$ are not real multiples of each other. Now the nullspace of $\tau=\pi_{1} \circ \psi$ is, on the one hand, a 1-real-dimensional subspace of $\mathbb{C}$, but on the other hand, it is all $E$-multiples of a fixed element $r_{2}$. Each $E$-multiple of $r_{2}$ is a real multiple, and vice versa. Divide by $r_{2}$ to conclude that $E=\mathbb{R}$.

Remarks. If $X \subseteq \mathbb{C}$ is an analytic set, then the ring generated by it, $\mathbb{Z}[X]$, is an analytic set. If $\operatorname{dim} X^{k}>0$, then $\mathbb{Z}[X]=\mathbb{R}$ or $\mathbb{Z}[X]=\mathbb{C}$. Of course $\mathbb{Z}[X]=\mathbb{R}$ or $\mathbb{C}$ according to whether $X \subseteq \mathbb{R}$ or not.

\section{SUBRINGS OF THE $p$-ADIC NUMBERS}

An analogous result may be proved for the fields of $p$-adic numbers.

Let $p$ be a prime, $\mathbb{Q}_{p}$ the complete metric locally compact field of $p$-adic numbers, and $\mathbb{Z}_{p}$ the compact subring of $p$-adic integers [19], [11]. The absolute value on $\mathbb{Q}_{p}$ is ultrametric and has only values 0 and $p^{n}$ for integers $n$. The Haar measure $\lambda$ is normalized so that $\lambda\left(\mathbb{Z}_{p}\right)=1$. Now $\mathbb{Z}_{p}$ is self-similar: it is the union of $p$ sets that are translates of $p \mathbb{Z}_{p}$, which is similar to $\mathbb{Z}_{p}$ itself, shrunk by the factor $p^{-1}$. So the Hausdorff dimension is the similarity dimension, namely 1. (Also the 1-dimensional Hausdorff measure is the Haar measure.)

Theorem 3. Let $E \subseteq \mathbb{Q}_{p}$ be a subring and a Borel set. Then $E$ has zero $C H$ dimension or $E=\mathbb{Q}_{p}$ or $E=\mathbb{Z}_{p}$.

The proof is essentially the same as before. We provide only a few remarks on the differences, and leave the details to the reader.

Lemma 3.1. Let $A \subseteq \mathbb{Q}_{p}^{k}$ be a Borel set with $\operatorname{dim} A>1$. Then for almost all linear functionals $\varphi: \mathbb{Q}_{p}^{k} \rightarrow \mathbb{Q}_{p}$, the image $\varphi(A)$ has positive Haar measure.

If $k$ is a positive integer, then the set $\mathbb{Q}_{p}^{k}$ of $k$-tuples is a vector space over $\mathbb{Q}_{p}$. The natural norm for it is defined as follows: if $\mathbf{v}=\left(v_{1}, \cdots, v_{k}\right)$, then

$$
\|\mathbf{v}\|=\max _{1 \leq j \leq k}\left|v_{j}\right| .
$$

Thus $\mathbb{Q}_{p}^{k}$ is again ultrametric, and the distances are all of the form $p^{n}$. Use the product measure $\lambda^{k}$ on $\mathbb{Q}_{p}^{k}$. For any $\mathbf{v} \in \mathbb{Q}_{p}^{k}$, define a linear functional $\varphi_{\mathbf{v}}: \mathbb{Q}_{p}^{k} \rightarrow \mathbb{Q}_{p}$ by

$$
\varphi_{\mathbf{v}}(\mathbf{u})=v_{1} u_{1}+\cdots+v_{k} u_{k}
$$

Therefore "almost all" linear functionals refers to the measure $\lambda^{k}$. For the unit sphere $S=\left\{\mathbf{v} \in \mathbb{Q}_{p}^{k}:\|\mathbf{v}\|=1\right\}$ there is no need for a surface area measure, since $S$ has positive $\lambda$ volume itself.

Lemma 3.2. Let $E \subseteq \mathbb{Q}_{p}$ be an additive subgroup and a Borel set with nonzero $C H$ dimension. Then there is a positive integer $k$ and a $\mathbb{Q}_{p}$-linear functional $\varphi: \mathbb{Q}_{p}^{k} \rightarrow$ $\mathbb{Q}_{p}$ such that $\varphi\left(E^{k}\right)$ is an open subgroup.

Use versions of the Dimension Inequality [5. (3.2.12), p. 122] and Steinhaus's Theorem [11, Cor. 20.17, p. 296] valid in this setting. The open subgroups of $\mathbb{Q}_{p}$ are $\mathbb{Q}_{p}$ itself and the multiples $p^{n} \mathbb{Z}_{p}$ of $\mathbb{Z}_{p}$. 
Lemma 3.3. Let $E \subseteq \mathbb{Q}_{p}$ be a subring. Assume there is a positive integer $k$ and a $\mathbb{Q}_{p}$-linear functional $\varphi: \mathbb{Q}_{p}^{k} \rightarrow \mathbb{Q}_{p}$ such that $\varphi\left(E^{k}\right)$ is an open subgroup. Then such $k$ and $\varphi$ may be found so that $\varphi$ also maps $E^{k}$ bijectively onto an open subgroup.

If $G$ is the open subgroup $\varphi\left(E^{k}\right)$, then proceed as before: The range for the restriction to $k-1$ coordinates is $b_{k} G$, also an open subgroup.

Lemma 3.4. Let $E \subseteq \mathbb{Q}_{p}$ be an additive subgroup and a Borel set. Let $k$ be a positive integer and $\varphi: \mathbb{Q}_{p}^{k} \rightarrow \mathbb{Q}_{p}$ a $\mathbb{Q}_{p}$-linear functional. Assume that $\varphi$ maps $E^{k}$ bijectively onto an open subgroup. Then $k=1$ and $E$ is an open subgroup.

Automatic continuity (a Borel-measurable homomorphism is continuous) is valid in any complete metric group: [2, Ch. I, Thm. 4], [14, (9.10), p. 61], [12, 2.3.1, p. 350].

Finally, if $K$ is a finite algebraic extension field of $\mathbb{Q}_{p}$ and $E \subseteq K$ is a subring and a Borel set, then $E$ has zero $\mathrm{CH}$ dimension or $E$ is a closed subring. The proofs are similar again. In an $n$-dimensional extension of $\mathbb{Q}_{p}$ the unit ball is self-similar, has Hausdorff dimension $n$, and $n$-dimensional Haar measure coincides with Hausdorff measure.

According to the classification in 19$], \mathbb{R}, \mathbb{C}$, and the finite algebraic extensions of the $\mathbb{Q}_{p}$ are all of the nondiscrete locally compact commutative fields of characteristic 0 .

\section{ADDED AFTER POSTING}

Change "additive subgroup" to "subring" in the statements of Lemmas 1.4, 2.4, and 3.4 .

\section{REFERENCES}

[1] C. Aliprantis and O. Burkinshaw, Principles of real analysis, 1st ed., North-Holland, New York, 1981. MR 82j:28001

[2] S. Banach, Théorie des opérations linéaires, Państwowe Wydawnictwo Naukowe, Warsaw, 1932. MR 97d:01035

[3] D. Cohn, Measure theory, Birkhäuser, Boston, 1980. MR 81k:28001

[4] R. Davies, Subsets of finite measure in analytic sets, Indag. Math. 14 (1952), 488-489. MR 14:733g

[5] G. Edgar, Integral, probability and fractal measure, Springer-Verlag, New York, 1998. MR 99c:28024

[6] G. Edgar and C. Miller, Hausdorff dimension, analytic sets and transcendence, Real Anal. Exchange, 27 (2001/02), 335-339.

[7] P. Erdős and B. Volkmann, Additive Gruppen mit vorgegebener Hausdorffscher Dimension, J. Reine Angew. Math. 221 (1966), 203-208. MR 32:4238

[8] K. Falconer, Rings of fractional dimension, Mathematika 31 (1984), 25-27. MR 85m:28004

[9] - On the Hausdorff dimensions of distance sets, Mathematika 32 (1985), 206-212. MR 87j:28008

[10] _ Fractal geometry: Mathematical foundations and applications, John Wiley \& Sons, Chichester, 1990. MR 92j:28008

[11] E. Hewitt and K. Ross, Abstract harmonic analysis, vol. I, Springer-Verlag, New York, 1963. MR 28:158

[12] F. Topsøe and J. Hoffmann-Jørgensen, Analytic spaces and their application, Analytic Sets, Academic Press, London, 1980, pp. 317-401.

[13] J. Howroyd, On dimension and on the existence of sets of finite positive Hausdorff measure, Proc. London Math. Soc. 70 (1995), 581-604. MR 96b:28004

[14] A. Kechris, Classical descriptive set theory, Grad. Texts Math., vol. 156, Springer-Verlag, 1995. MR 96e:03057 
[15] P. Mattila, Geometry of sets and measures in euclidean spaces, Cambridge Stud. Adv. Math., vol. 44, Cambridge Univ. Press, Cambridge, 1995. MR 96h:28006

[16] K. Stromberg, An introduction to classical real analysis, Wadsworth, Belmont, CA, 1981. MR 82c:26002

[17] B. Volkmann, Eine metrische Eigenschaft reeler Zahlkörper, Math. Ann. 141 (1960), 237238. MR 22:8097

[18] H. Wegmann, Die Hausdorff-Dimension von kartesischen Produkten matrischer Räume, J. Reine Angew. Math. 246 (1971), 46-75. MR 42:8463

[19] A. Weil, Basic number theory, Springer-Verlag, New York, 1967. MR 38:3244

Department of Mathematics, The Ohio State University, 231 West Eighteenth Avenue, Columbus, Ohio 43210

E-mail address: edgar@math.ohio-state.edu

Department of Mathematics, The Ohio State University, 231 West Eighteenth Avenue, Columbus, Ohio 43210

E-mail address: miller@math.ohio-state.edu 